\title{
Summation of rational series twisted by strongly $B$-multiplicative coefficients
}

\author{
Jean-Paul Allouche* \\ CNRS, Institut de Mathématiques de Jussieu-PRG \\ Université Pierre et Marie Curie, Case 247 \\ F-75252 Paris Cedex 05, France \\ jean-paul .allouche@imj-prg.fr
}

\author{
Jonathan Sondow \\ 209 West 97 th Street \\ New York \\ NY 10025, U.S.A \\ jsondow@alumni.princeton.edu
}

Submitted: Aug 26, 2014; Accepted: Feb 17, 2015; Published: Mar 6, 2015

Mathematics Subject Classifications: 11A63, 11B83, 11B85, 68R15, 05A19

\begin{abstract}
We evaluate in closed form series of the type $\sum u(n) R(n)$, with $(u(n))_{n}$ a strongly $B$-multiplicative sequence and $R(n)$ a (well-chosen) rational function. A typical example is:

$$
\sum_{n \geqslant 1}(-1)^{s_{2}(n)} \frac{4 n+1}{2 n(2 n+1)(2 n+2)}=-\frac{1}{4}
$$

where $s_{2}(n)$ is the sum of the binary digits of the integer $n$. Furthermore closed formulas for series involving automatic sequences that are not strongly $B$-multiplicative, such as the regular paperfolding and Golay-Shapiro-Rudin sequences, are obtained; for example, for integer $d \geqslant 0$ :

$$
\sum_{n \geqslant 0} \frac{v(n)}{(n+1)^{2 d+1}}=\frac{\pi^{2 d+1}\left|E_{2 d}\right|}{\left(2^{2 d+2}-2\right)(2 d) !}
$$

where $(v(n))_{n}$ is the \pm 1 regular paperfolding sequence and $E_{2 d}$ is an Euler number.

Keywords: summation of series; strongly $B$-multiplicative sequences; paperfolding sequence; Golay-Shapiro-Rudin sequence
\end{abstract}

\section{Introduction}

The problem of evaluating a series $\sum_{n} R(n)$ where $R$ is a rational function with integer coefficients is classical: think of the values of the Riemann $\zeta$ function at integers. Such

\footnotetext{
*The author was partially supported by the ANR project "FAN" (Fractals et Numération), ANR-12IS01-0002.
} 
sums can also be "twisted", usually by a character (think of the $L$-functions), or by the usual arithmetic functions (e.g., the Möbius function $\mu$ ).

Another possibility is to twist such sums by sequences related to the digits of $n$ in some integer base. Examples can be found in [5] with, in particular, series $\sum \frac{u(n)}{n(n+1)}$, and in [7] with, in particular, series $\sum \frac{u(n)}{2 n(2 n+1)}$ (also see [9]): in both cases $u(n)$ counts the number of occurrences of a given block of digits in the $B$-ary expansion of the integer $n$, or is equal to $s_{B}(n)$, the sum of the $B$-ary digits of the integer $n$ ( $B$ being an integer $\geqslant 2$ ). Two emblematic examples are (see [10, Problem B5, p. 682] and [12, 5] for the first one, and $[14,7]$ for the second one):

$$
\sum_{n \geqslant 1} \frac{s_{B}(n)}{n(n+1)}=\frac{B}{B-1} \text { and } \sum_{n \geqslant 1} \frac{s_{2}(n)}{2 n(2 n+1)}=\frac{\gamma+\log \frac{4}{\pi}}{2}
$$

where $\gamma$ is the Euler-Mascheroni constant.

Similarly one can try to evaluate infinite products $\prod_{n} R(n)$, where $R(n)$ is a rational function, as well as twisted such products $\prod_{n} R(n)^{u(n)}$, where the sequence $(u(n))_{n \geqslant 0}$ is related to the digits of $n$ in some integer base. An example can be found in [2] (also see [11] for the original problem):

$$
\prod_{n \geqslant 1}\left(\frac{(4 n+2)(4 n+2)}{(4 n+1)(4 n+3)}\right)^{2 z(n)}=\frac{4}{\pi}
$$

where $z(n)$ is the sum of the number of 0 's and the number of 1's in the binary expansion of $n$, i.e., the length of this expansion. Other examples can be found in [4], e.g.,

$$
\prod_{n \geqslant 0}\left(\frac{(4 n+2)(8 n+7)(8 n+3)(16 n+10)}{(4 n+3)(8 n+6)(8 n+2)(16 n+11)}\right)^{u(n)}=\frac{1}{\sqrt{2}}
$$

where $u(n)=(-1)^{a(n)}$ and $a(n)$ is equal to the number of blocks 1010 occurring in the binary expansion of $n$. The products studied in [4] (also see references therein) are of the form $\prod_{n} R(n)^{(-1)^{a(n)}}$ where $R(n)$ is a (well-chosen) rational function with integer coefficients, and $a(n)$ counts the number of occurrences of a given block of digits in the $B$-ary expansion of the integer $n$. The case where $a(n)$ counts the number of 1 's occurring in the binary expansion of $n$ is nothing but the case $a(n)=s_{2}(n)$. If $a(n)=s_{B}(n)$, the sequence $\left((-1)^{a(n)}\right)_{n \geqslant 0}$ is strongly $B$-multiplicative: the more general evaluation of the product $\prod_{n} R(n)^{u(n)}$ where $(u(n))_{n \geqslant 0}$ is a strongly $B$-multiplicative sequence, is addressed in [8] (also see [13]). Recall that a strongly $B$-multiplicative sequence $(u(n))_{n \geqslant 0}$ satisfies $u(0)=0$, and $u(B n+j)=u(n) u(j)$ for all $j \in[0, B-1]$ and all $n \geqslant 0$. In particular, $(u(n))_{n \geqslant 0}$ is $B$-regular (or even $B$-automatic if it takes only finitely many values): recall that a sequence $(u(n))_{n \geqslant 0}$ is called $B$-automatic if its $B$-kernel, i.e., the set of subsequences $\left\{\left(u\left(B^{a} n+r\right)\right)_{n \geqslant 0} \mid a \geqslant 0,0 \leqslant r \leqslant B^{a}-1\right\}$, is finite; a sequence $(u(n))_{n \geqslant 0}$ with values 
in $\mathbb{Z}$ is called $B$-regular if the $\mathbb{Z}$-module spanned by its $B$-kernel has finite type (for more on these notions, see, e.g., [6]).

Since $\log \prod_{n} R(n)^{u(n)}=\sum_{n} u(n) \log R(n)$, it is natural to look at "simpler" series of the form $\sum_{n} u(n) R(n)$ with $R$ and $u$ as previously. All the examples above involve sequences $(u(n))_{n \geqslant 0}$ that are $B$-regular or even $B$-automatic. Unfortunately we were not able to address the general case where $(u(n))_{n \geqslant 0}$ is any $B$-regular or any $B$-automatic sequence. The purpose of the present paper is to study the special case where, as in [8], the sequence $u(n)$ is strongly $B$-multiplicative and $R(n)$ is a well-chosen rational function. The paper can thus be seen as a companion paper to [8]. We will end with the evaluation of similar series where $(u(n))_{n \geqslant 0}$ is the regular paperfolding sequence or the Golay-Shapiro-Rudin sequence.

\section{Preliminary definitions and results}

This section quickly recalls definitions and results from [8].

Definition 1. Let $B \geqslant 2$ be an integer. A sequence of complex numbers $(u(n))_{n \geqslant 0}$ is strongly $B$-multiplicative if $u(0)=1$ and, for all $n \geqslant 0$ and all $k \in\{0,1, \ldots, B-1\}$,

$$
u(B n+k)=u(n) u(k) .
$$

Example 2. Let $B \geqslant 2$ be an integer and $s_{B}(n)$ be the sum of the $B$-ary digits of $n$. Then for every complex number $a \neq 0$ the sequence $\left(a^{s_{B}(n)}\right)_{n \geqslant 0}$ is strongly $B$-multiplicative. This sequence is $B$-regular (see the introduction); it is $B$-automatic if and only if $a$ is a root of unity.

The following lemma is a variation of Lemma 1 in [8].

Lemma 3. Let $B>1$ be an integer. Let $(u(n))_{n \geqslant 0}$ be a strongly $B$-multiplicative sequence of complex numbers different from the sequence $(1,0,0, \ldots)$. We suppose that $|u(n)| \leqslant 1$ for all $n \geqslant 0$ and that $\left|\sum_{0 \leqslant k<B} u(k)\right|<B$. Let $f$ be a map from the set of nonnegative integers to the set of complex numbers such that $|f(n+1)-f(n)|=\mathcal{O}\left(n^{-2}\right)$. Then the series $\sum_{n \geqslant 0} u(n) f(n)$ is convergent.

Proof. Use [8, Lemma 1] to get the upper bound $\left|\sum_{0 \leqslant n<N} u(n)\right|<C N^{\alpha}$ for some positive constant $C$ and some real number $\alpha$ in $(0,1)$. Then use summation by parts.

\section{Main results}

We state in this section some basic identities as well as first applications and examples. First we define $\delta_{k}$, a special case of the Kronecker delta:

$$
\delta_{k}= \begin{cases}1 & \text { if } k=0 \\ 0 & \text { otherwise }\end{cases}
$$


Theorem 4. Let $B>1$ be an integer. Let $(u(n))_{n \geqslant 0}$ be a strongly B-multiplicative sequence, and let $f$ be a map from the nonnegative integers to the complex numbers, such that $(u(n))_{n \geqslant 0}$ and $f$ satisfy the conditions of Lemma 3. Define the series $S_{1}(k, B, u, f)$, for $k=0,1, \ldots, B-1$, by

$$
S(k, B, u, f):=\sum_{n \geqslant 0} u(n) f(B n+k) .
$$

Then the following linear relations hold:

$$
\sum_{n \geqslant 0} u(n) f(n)=\sum_{0 \leqslant k \leqslant B-1} u(k) S(k, B, u, f)
$$

and

$$
\sum_{n \geqslant 0} u(n) \sum_{0 \leqslant k \leqslant B-1} f(B n+k)=\sum_{0 \leqslant k \leqslant B-1} S(k, B, u, f) .
$$

In particular, define the series $S_{1}(k, B, u)$ and $S_{2}(k, B, u)$, for $k=0,1, \ldots, B-1$, by

$$
S_{1}(k, B, u):=\sum_{n \geqslant \delta_{k}} \frac{u(n)}{B n+k} \text { and } S_{2}(k, B, u):=\sum_{n \geqslant \delta_{k}} \frac{u(n)}{(B n+k)(B n+k+1)} .
$$

Then the following linear relations hold:

$$
(B-1) S_{1}(0, B, u)-\sum_{1 \leqslant k \leqslant B-1} u(k) S_{1}(k, B, u)=0
$$

and

$$
\sum_{0 \leqslant k \leqslant B-1}(B-u(k)) S_{2}(k, B, u)=B-1 .
$$

Proof. It follows from Lemma 3 that all the series in the theorem converge. To prove the first relation, we split $\sum_{n \geqslant 0} u(n) f(n)$, obtaining

$$
\begin{aligned}
\sum_{n \geqslant 0} u(n) f(n) & =\sum_{0 \leqslant k \leqslant B-1} \sum_{n \geqslant 0} u(B n+k) f(B n+k)=\sum_{0 \leqslant k \leqslant B-1} \sum_{n \geqslant 0} u(n) u(k) f(B n+k) \\
& =\sum_{0 \leqslant B-1} u(k) \sum_{n \geqslant 0} u(n) f(B n+k)=\sum_{0 \leqslant k \leqslant B-1} u(k) S(k, B, u, f) .
\end{aligned}
$$

To prove the second relation, we write

$$
\sum_{n \geqslant 0} u(n) \sum_{0 \leqslant k \leqslant B-1} f(B n+k)=\sum_{0 \leqslant k \leqslant B-1} \sum_{n \geqslant 0} u(n) f(B n+k)=\sum_{0 \leqslant k \leqslant B-1} S(k, B, u, f) .
$$

To prove the last part of the theorem, we make two choices for $f$. First we take $f$ defined by $f(n)=1 / n$ for $n \neq 0$ and $f(0)=0$. Then we take $f(n)=1 / n(n+1)$ if $n \neq 0$ and $f(0)=0$. 
Remark The formula $S_{2}(k, B, u)=S_{1}(k, B, u)-\left(S_{1}(k+1, B, u)-\delta_{k}\right)(0 \leqslant k \leqslant B-2)$ holds. Nevertheless, the last two relations in Theorem 4 are independent, because $S_{2}(B-$ $1, B, u)$ cannot be expressed in terms of the $S_{1}(k, B, u)$ for $k=0,1, \ldots, B-1$.

Corollary 5. If $(u(n))_{n \geqslant 0}$ is a strongly $B$-multiplicative sequence satisfying the conditions of Lemma 3, then

$$
\sum_{n \geqslant 1} u(n) \sum_{1 \leqslant k \leqslant B-1}\left(\frac{1}{B n}-\frac{u(k)}{B n+k}\right)=\sum_{1 \leqslant k \leqslant B-1} \frac{u(k)}{k}
$$

and

$$
\sum_{n \geqslant 1} u(n) \sum_{0 \leqslant k \leqslant B-1} \frac{B-u(k)}{(B n+k)(B n+k+1)}=\sum_{1 \leqslant k \leqslant B-1} \frac{u(k)}{k(k+1)} .
$$

Proof. This follows from the last part of Theorem 4 by substitution and manipulation.

Recall that the $n$th harmonic number $H_{n}$ and the $n$th alternating harmonic number $H_{n}^{*}$ are defined by

$$
H_{n}:=\sum_{1 \leqslant k \leqslant n} \frac{1}{k} \text { and } H_{n}^{*}:=\sum_{1 \leqslant k \leqslant n} \frac{(-1)^{k-1}}{k} .
$$

Corollary 6. If $N_{j, B}(n)$ is the number of occurrences of the digit $j \in\{0,1, \ldots, B-1\}$ in the $B$-ary expansion of $n$, then the following summations hold when $j \neq 0$ :

$$
\sum_{n \geqslant 1}(-1)^{N_{j, B}(n)}\left(\frac{2}{B n+j}+\frac{1}{B n} \sum_{1 \leqslant k \leqslant B-1} \frac{k}{B n+k}\right)=H_{B-1}-\frac{2}{j}
$$

and

$$
\sum_{n \geqslant 1}(-1)^{N_{j, B}(n)}\left(\frac{B-1}{n(n+1)}+\frac{2 B}{(B n+j)(B n+j+1)}\right)=B-1-\frac{2 B}{j(j+1)} .
$$

Proof. It is not hard to see that, if $j \neq 0$, we can apply the last part of Theorem 4 to the sequence $u(n):=(-1)^{N_{j, B}(n)}$. Using Corollary 5 and the fact that $N_{j, B}(k)=\delta_{k, j}$ when $0 \leqslant k<B$, the result follows.

Example 7. Taking $B=2$ and $j=1$, we get

$$
\sum_{n \geqslant 1}(-1)^{N_{1,2}(n)} \frac{4 n+1}{2 n(2 n+1)}=-1
$$

and

$$
\sum_{n \geqslant 1}(-1)^{N_{1,2}(n)} \frac{4 n+1}{2 n(2 n+1)(2 n+2)}=-\frac{1}{4} .
$$


Subtracting the second equation from the first, we multiply by 4 and obtain

$$
\sum_{n \geqslant 1}(-1)^{N_{1,2}(n)} \frac{4 n+1}{n(n+1)}=-3 .
$$

With $B=3$ and $j=1$ we get

$$
\sum_{n \geqslant 1}(-1)^{N_{1,3}(n)} \frac{18 n^{2}+21 n+4}{3 n(3 n+1)(3 n+2)}=-\frac{1}{2}
$$

and

$$
\sum_{n \geqslant 1}(-1)^{N_{1,3}(n)} \frac{6 n^{2}+6 n+1}{3 n(3 n+1)(3 n+2)(3 n+3)}=-\frac{1}{36} .
$$

Corollary 8. If $s_{B}(n)$ is the sum of the B-ary digits of $n$, then

$$
\sum_{n \geqslant 1}(-1)^{s_{B}(n)} \sum_{1 \leqslant k \leqslant B-1}\left(\frac{1}{B n}-\frac{(-1)^{k}}{B n+k}\right)=-H_{B-1}^{*}
$$

and

$$
\sum_{n \geqslant 1}(-1)^{s_{B}(n)} \sum_{0 \leqslant k \leqslant B-1} \frac{B-(-1)^{k}}{(B n+k)(B n+k+1)}=1+\frac{(-1)^{B}}{B}-2 H_{B-1}^{*} .
$$

Proof. Setting $u(n):=(-1)^{s_{B}(n)}$, it is not hard to see that $u(2 n+1)=-u(2 n)$ for all $n \geqslant 0$. (Hint: look at the cases $B$ even and $B$ odd separately.) It follows that $(u(n))_{n \geqslant 0}$ satisfies the conditions of Lemma 3. Noting that $u(k)=(-1)^{k}$ when $0 \leqslant k<B$, the result follows from Corollary 5 .

Example 9. Taking $B=2$ or 3 gives the same pair of series as those with that value of $B$ in Example 1, since $s_{2}(n)=N_{1,2}(n)$ and $s_{3}(n)=N_{1,3}(n)+2 N_{2,3}(n)$. (We can also replace $s_{3}(n)$ with $n$, as $(-1)^{s_{B}(n)}=(-1)^{n}$ when $B$ is odd.) With $B=4$ we get

$$
\sum_{n \geqslant 1}(-1)^{s_{4}(n)} \frac{128 n^{3}+176 n^{2}+76 n+9}{4 n(4 n+1)(4 n+2)(4 n+3)}=-\frac{5}{12}
$$

and

$$
\sum_{n \geqslant 1}(-1)^{s_{4}(n)} \frac{128 n^{3}+184 n^{2}+80 n+9}{4 n(4 n+1)(4 n+2)(4 n+3)(4 n+4)}=-\frac{5}{12}
$$

\section{More examples}

Using Corollary 5 with sequences $(u(n))_{n \geqslant 0}$ taking complex values yields other examples of sums of series. 
Example 10. We may let $u(n):=i^{s_{2}(n)}$ in Corollary 5. This gives the two summations

$$
\sum_{n \geqslant 1}\left(\frac{i^{s_{2}(n)}}{2 n}-\frac{i^{s_{2}(n)+1}}{2 n+1}\right)=i=\sum_{n \geqslant 1} \frac{i^{s_{2}(n)}(3 n+1)-i^{s_{2}(n)+1} n}{n(n+1)(2 n+1)},
$$

and by taking the imaginary and real parts we obtain the following result:

If $\chi$ is the non-principal Dirichlet character modulo 4, defined by

$$
\chi(n):=\left\{\begin{aligned}
+1 & \text { if } n \equiv 1 \bmod 4 \\
-1 & \text { if } n \equiv 3 \bmod 4 \\
0 & \text { otherwise }
\end{aligned}\right.
$$

then

$$
\sum_{n \geqslant 1}\left(\frac{\chi\left(s_{2}(n)\right)}{2 n}-\frac{\chi\left(s_{2}(n)+1\right)}{2 n+1}\right)=1=\sum_{n \geqslant 1} \frac{(3 n+1) \chi\left(s_{2}(n)\right)-n \chi\left(s_{2}(n)+1\right)}{n(n+1)(2 n+1)}
$$

and

$$
\sum_{n \geqslant 1}\left(\frac{\chi\left(s_{2}(n)+1\right)}{2 n}-\frac{\chi\left(s_{2}(n)+2\right)}{2 n+1}\right)=0=\sum_{n \geqslant 1} \frac{(3 n+1) \chi\left(s_{2}(n)+1\right)-n \chi\left(s_{2}(n)+2\right)}{n(n+1)(2 n+1)} .
$$

Example 11. Generalizing Example 10 by replacing $i^{s_{2}(n)}$ with $e^{2 i \pi s_{2}(n) / d}$, for integer $d \geqslant 2$, is straightforward, yielding the following summations (Example 10 is another formulation for the case $d=4)$ :

$$
\sum_{n \geqslant 1}\left(\frac{\sin \frac{2 \pi s_{2}(n)}{d}}{2 n}-\frac{\sin \frac{2 \pi\left(s_{2}(n)+1\right)}{d}}{2 n+1}\right)=\sin \frac{2 \pi}{d}=\sum_{n \geqslant 1} \frac{(3 n+1) \sin \frac{2 \pi s_{2}(n)}{d}-n \sin \frac{2 \pi\left(s_{2}(n)+1\right)}{d}}{n(n+1)(2 n+1)}
$$

and

$$
\sum_{n \geqslant 1}\left(\frac{\cos \frac{2 \pi s_{2}(n)}{d}}{2 n}-\frac{\cos \frac{2 \pi\left(s_{2}(n)+1\right)}{d}}{2 n+1}\right)=\cos \frac{2 \pi}{d}=\sum_{n \geqslant 1} \frac{(3 n+1) \cos \frac{2 \pi s_{2}(n)}{d}-n \cos \frac{2 \pi\left(s_{2}(n)+1\right)}{d}}{n(n+1)(2 n+1)} .
$$

\section{The paperfolding and Golay-Shapiro-Rudin sequences}

The results above involve sums $\sum u(n) R(n)$ where $(u(n))_{n \geqslant 0}$ is a strongly $B$-multiplicative sequence, which, in all of our examples except Example 2 with alpha not a root of unity, happens to take only finitely many values. This implies that $(u(n))_{n \geqslant 0}$ is $B$-automatic (see the introduction). One can then ask about more general sums $\sum u(n) R(n)$ where the sequence $(u(n))_{n \geqslant 0}$ is $B$-automatic. We give two cases where such series can be summed. 
Theorem 12. Let $(v(n))_{n \geqslant 0}$ be the regular paperfolding sequence. Its first few terms are given by (replacing +1 by + and -1 by - )

$$
(v(n))_{n \geqslant 0}=++-++-\ldots ;
$$

it can be defined by: $v(2 n)=(-1)^{n}$ and $v(2 n+1)=v(n)$ for all $n \geqslant 0$. Then, for all integers $d \geqslant 0$, we have the relation

$$
\sum_{n \geqslant 0} \frac{v(n)}{(n+1)^{2 d+1}}=\frac{\pi^{2 d+1}\left|E_{2 d}\right|}{\left(2^{2 d+2}-2\right)(2 d) !}
$$

where the $E_{2 d}$ 's are the Euler numbers defined by:

$$
\frac{1}{\cosh t}=\sum_{n \geqslant 0} \frac{E_{2 n}}{(2 n) !} t^{2 n} \text { for }|t|<\frac{\pi}{2} .
$$

Proof. First note that the series $\sum_{n \geqslant 0} \frac{v(n)}{(n+1)^{s}}$ converges for $\Re(s)>0$ : use the inequality $\left|\sum_{n<N} v(n)\right|=O(\log N)$ (see, e.g., [6, Exercise 28, p. 206]) and summation by parts; note that the sequence $\left(R_{n}\right)_{n \geqslant 1}$ in $\left[6\right.$, Exercise 28, p. 206] is equal to the sequence $(v(n))_{n \geqslant 0}$ here. Now, Exercise 27 in [6, p. 205-206] asks to prove, for all complex numbers $s$ with $\Re(s)>0$, the equality (again with slightly different notation)

$$
\sum_{n \geqslant 0} \frac{v(n)}{(n+1)^{s}}=\frac{2^{s}}{2^{s}-1} \sum_{n \geqslant 0} \frac{(-1)^{n}}{(2 n+1)^{s}} .
$$

This can be easily done by splitting the sum on the left into even and odd indexes. Recalling that the Dirichlet beta function is defined by $\beta(s)=\sum_{n \geqslant 0} \frac{(-1)^{n}}{(2 n+1)^{s}}$ for $\Re(s)>0$, we thus have, for any nonnegative integer $d$,

$$
\sum_{n \geqslant 0} \frac{v(n)}{(n+1)^{2 d+1}}=\frac{2^{2 d+1}}{2^{2 d+1}-1} \beta(2 d+1) .
$$

But, when $s$ is an odd integer, the value of $\beta(s)$ can be expressed as a rational multiple of $\pi$ (see, e.g., [1, 23.2.22, p. 807]):

$$
\beta(2 d+1)=\frac{(\pi / 2)^{2 d+1}}{2(2 d) !}\left|E_{2 d}\right|
$$

Example 13. Taking $d=0$ in Theorem 12 yields a result due to F. von Haeseler (see $[6$, Exercise 27, p. 205-206])

$$
\sum_{n \geqslant 0} \frac{v(n)}{n+1}=\frac{\pi}{2}
$$

The second result we give in this section involves the Golay-Shapiro-Rudin sequence. 
Theorem 14. Let $(r(n))_{n \geqslant 0}$ be the \pm 1 Golay-Shapiro-Rudin sequence. This sequence can be defined by $r(n)=(-1)^{a(n)}$, where $a(n)$ is the number of possibly overlapping occurrences of the block 11 in the binary expansion of $n$, so that (replacing +1 by + and -1 by -1$)$

$$
(r(n))_{n \geqslant 0}=+++-++-+\ldots ;
$$

alternatively it can be defined by

$$
r(0)=1, \text { and } r(2 n)=r(n), r(2 n+1)=(-1)^{n} r(n) \text { for } n \geqslant 0 .
$$

Let $R(n)$ be a function from the nonnegative integers to the complex numbers, such that $|R(n+1)-R(n)|=\mathcal{O}\left(n^{-2}\right)$. Then we have the relation

$$
\sum_{n \geqslant 1} r(n)(R(n)-R(2 n)+R(2 n+1)-2 R(4 n+1))=R(1) .
$$

Proof. It is well known that $\left|\sum_{n<N} r(n)\right|<K \sqrt{n}$ for some positive constant $K$ (actually more is known; see, e.g., [6, Theorem 3.3.2, p. 79] and the historical comments given in $\left[6,3.3\right.$, p. 121]). Thus, by summation by parts, the series $\sum_{n \geqslant 0} r(n) R(n)$ is convergent. Now we write

$$
\begin{aligned}
\sum_{n \geqslant 0} r(n) R(n) & =\sum_{n \geqslant 0} r(2 n) R(2 n)+\sum_{n \geqslant 0} r(2 n+1) R(2 n+1) \\
& =\sum_{n \geqslant 0} r(n) R(2 n)+\sum_{n \geqslant 0}(-1)^{n} r(n) R(2 n+1) \\
& =\sum_{n \geqslant 0}^{n} r(n) R(2 n)+\sum_{n \geqslant 0} r(2 n) R(4 n+1)-\sum_{n \geqslant 0} r(2 n+1) R(4 n+3) \\
& =\sum_{n \geqslant 0} r(n)(R(2 n)+R(4 n+1))-\sum_{n \geqslant 0} r(2 n+1) R(4 n+3) .
\end{aligned}
$$

Hence

$$
\begin{aligned}
\sum_{n \geqslant 0} r(n)(R(n)-R(2 n)-R(4 n+1)) & =-\sum_{n \geqslant 0} r(2 n+1) R(4 n+3) \\
& =-\left(\sum_{n \geqslant 0}^{n} r(n) R(2 n+1)-\sum_{n \geqslant 0} r(2 n) R(4 n+1)\right) \\
& =-\sum_{n \geqslant 0}^{n} r(n) R(2 n+1)+\sum_{n \geqslant 0} r(n) R(4 n+1)
\end{aligned}
$$

where the penultimate equality is obtained by splitting the sum $\sum_{n \geqslant 0} r(n) R(2 n+1)$ into even and odd indices. Thus, finally

$$
\sum_{n \geqslant 0} r(n)(R(n)-R(2 n)+R(2 n+1)-2 R(4 n+1))=0
$$

hence

$$
\sum_{n \geqslant 1} r(n)(R(n)-R(2 n)+R(2 n+1)-2 R(4 n+1))=R(1) .
$$


Example 15. Taking $R(n)=1 / n$ if $n \neq 0$ and $R(0)=1$ in Theorem 14 above yields

$$
\sum_{n \geqslant 1} r(n) \frac{8 n^{2}+4 n+1}{2 n(2 n+1)(4 n+1)}=1 .
$$

Example 16. Taking $R$ defined by $R(n)=\log n-\log (n+1)$ for $n \neq 0$ and $R(0)=0$ in Theorem 14 above yields

$$
\sum_{n \geqslant 1} r(n) \log \frac{(2 n+1)^{4}}{(n+1)^{2}(4 n+1)^{2}}=-\log 2 .
$$

Hence

$$
\sum_{n \geqslant 0} r(n) \log \frac{(2 n+1)^{2}}{(n+1)(4 n+1)}=-\frac{1}{2} \log 2 .
$$

After exponentiating we obtain:

$$
\prod_{n \geqslant 0}\left(\frac{(2 n+1)^{2}}{(n+1)(4 n+1)}\right)^{r(n)}=\frac{1}{\sqrt{2}}
$$

thus recovering the value of an infinite product obtained in [3, Theorem 2, p. 148] (also see [4]).

\section{References}

[1] M. Abramowitz and I. A. Stegun. Handbook of Mathematical Functions With Formulas, Graphs, and Mathematical Tables, National Bureau of Standards Applied Mathematics Series, no 55, 10th printing, 1972.

[2] J.-P. Allouche. On a formula of T. Rivoal. Ann. Univ. Sci. Budapest. Sect. Comput., 40:69-79, 2013.

[3] J.-P. Allouche, H. Cohen, M. Mendès France, and J. O. Shallit. De nouveaux curieux produits infinis. Acta Arith., 49:141-153, 1987.

[4] J.-P. Allouche and J. O. Shallit. Infinite products associated with counting blocks in binary strings. J. London Math. Soc., 39:193-204, 1989.

[5] J.-P. Allouche and J. Shallit. Sums of digits and the Hurwitz zeta function. In Analytic number theory (Tokyo, 1988), volume 1434 of Lecture Notes in Math., pages 19-30. Springer, Berlin, 1990.

[6] J.-P. Allouche and J. Shallit. Automatic Sequences. Theory, Applications, Generalizations. Cambridge University Press, Cambridge, 2003.

[7] J.-P. Allouche, J. Shallit, and J. Sondow. Summation of series defined by counting blocks of digits. J. Number Theory, 123:133-143, 2007. 
[8] J.-P. Allouche and J. Sondow. Infinite products with strongly $B$-multiplicative exponents. Ann. Univ. Sci. Budapest. Sect. Comput., 28:35-53, 2008.

Also see J.-P. Allouche and J. Sondow. Errata for the paper J.-P. Allouche and J. Sondow: Infinite products with strongly B-multiplicative exponents. Ann. Univ. Sci. Budapest. Sect. Comput., 32:253, 2010.

[9] K. Hessami Pilehrood and T. Hessami Pilehrood. Vacca-type series for values of the generalized Euler constant function and its derivative. J. Integer Seq. 13(7):Article 10.7.3, 21 pp., 2010.

[10] L. F. Klosinski, G. L. Alexanderson, and A. P. Hillman. The William Lowell Putnam mathematical competition. Amer. Math. Monthly, 89:679-686, 1982.

[11] T. Rivoal. Polynômes de type Legendre et approximations de la constante d'Euler. Unpublished preprint (2005). http://www-fourier.ujf-grenoble.fr/ rivoal/ articles/euler.pdf

[12] J. O. Shallit. Two series, solution to Advanced Problem 6450 in Amer. Math. Monthly 91 (1984) 59-60. Amer. Math. Monthly, 92:513-514, 1985.

[13] J. Sondow. An infinite product based on a base, solution to Problem 11222 in Amer. Math. Monthly 113 (2006) 459. Amer. Math. Monthly, 115:954-955, 2008.

[14] J. Sondow. New Vacca-type rational series for Euler's constant and its "alternating" analog $\ln 4 / \pi$. In Additive number theory, Festschrift in honor of the sixtieth birthday of Melvyn B. Nathanson, pages 331-340. Eds., D. Chudnovsky, G. Chudnovsky, Springer, New York, 2010. 